\title{
A Research on the Extraction and Interpretation of Power Line Communication Noise Pattern Using Genetic Algorithm
}

\author{
Chung-Ki Seo ${ }^{1,2, a}$, Su-Mi Jeong ${ }^{1,2, b}$ and Duck-Jin Chung ${ }^{1, c}$ \\ ${ }^{1}$ Information Technology and Telecommunications, INHA University, \\ 253 Younghyun-Dong, Nam-Gu, Incheon 402-751, Korea \\ ${ }^{2}$ Dept. of Embedded Research, KEPCO KDN, \\ Naeson 2-Dong, Uiwang-Si, Gyeonggi-Do 437-715, Korea \\ ackseo@inha.edu, ${ }^{b}$ sm3010@kdn.com, djchung@inha.ac.kr
}

Keywords: smart grid, advanced metering infrastructure, auto meter reading, data concentrate unit, power line communication, noise, pattern recognition

\begin{abstract}
A PLC is more sensitive in noise than other wired communication system that is cable, DSL, and optical LAN. Therefore it considered as a crucial point in commercialization of PLC. For the commercialization of PLC, noise reduction and cancelation technique are needed. Therefore, it is definitely required to analyze and interpret the effects and types of the noise, exactly. The purpose of this work is to separate and extract the various noise of power line from data signals.
\end{abstract}

\section{Introduction}

The AMR-based AMI system uses PLC as a main communication technology to build Smart Grid. Despite these advantages, PLC is quite weak in the inflow noise of power lines because it uses deteriorated power lines, low-frequency band $(1 \sim 30 \mathrm{MHz})$ compared to other wired communication, and same frequency band for both upward and downward communication.[2] The weakness in noise decreases its stability and reliability and it is the significant barrier of PLC diffusion. For this reason, the research for minimizing and eliminating the critical effect of noise has been driven because it decreases performance of PLC. This paper introduces the study for recognizing and classifying the PLC noise pattern to minimize the critical effect of PLC noise with GA(Genetic Algorithm).

\section{The PLC noise of AMR}

In Korea, KEPCO(Korea Electric Power Corporation) consortium is mainly establishing and providing the AMR-based AMI system and the related service actively. For several decades, we spurring the development of new system to perform the remote meter reading for most consumers using low voltage. The bi-directional PLC technology is used between DCU and a smart meter, between DCU and gateway. It uses the power line inside of consumer's residence in the configuration of network, therefore it is quite weak in the noise caused by deteriorated power lines and electronic products. There are several kinds of noise such as (1) colored background noise; (2) narrow band noise; (3) periodic impulse noise synchronous with power frequency; (4) non-periodic impulse noise; (5) burst noise.[3] Of these, we simulated pattern recognition to discriminate the color noise occurs in a specific frequency band. The configuration of AMR operated currently is shown in Fig. 1.

\section{GA for extracting PLC noise characteristic pattern}

Recently, the large-scale optimization problems with complex constraints among various engineering applications are very difficult to get optimal solutions with general mathematical programs and a combination of optimization algorithm within a short computation time. Therefore, GA which is one of the evolutionary algorithms is used as the primary mechanism for solving these problems within a reasonable computation time.[4] GA finds a candidate solution with selection, crossover and mutation for the problem and it also finds an optimal solution by transforming population which is a set of organisms into new population repeatedly. These are basic operations of the GA to define a mechanism and improve its efficiency. [5] The basic algorithm flow chart is shown in Fig. 2. 


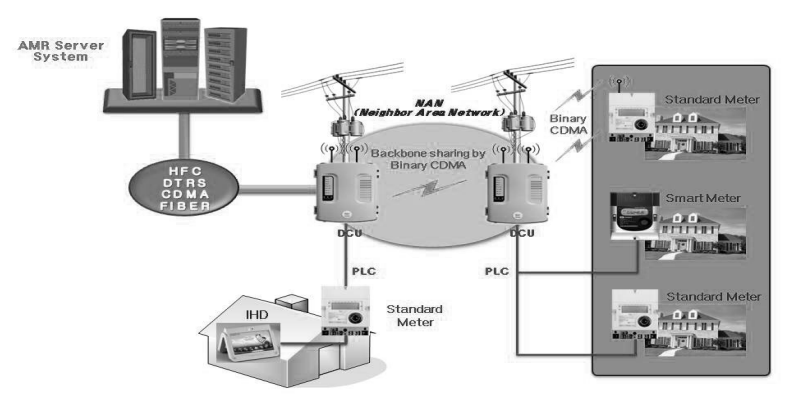

Fig. 1. The PLC configuration based on the AMR

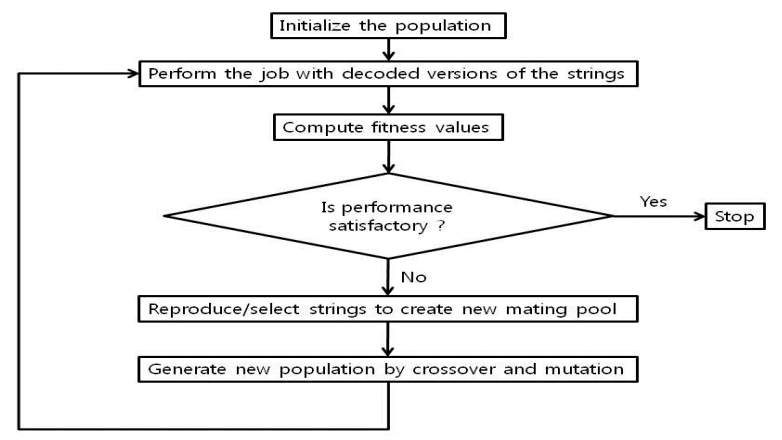

Fig. 2. Basic flow chart of a GA

We implemented the algorithm based on the basic GA that will be applied to the PLC noise pattern recognition. (Algorithm. 1) The arithmetic expressions applied to the Algorithm. 1 are defined as follows Eq. 1 to Eq. 6

Algorithm 1. A Genetic Algorithm with proposed pattern recognition

procedure $\mathrm{GA}()$ initialize(Population); evaluate(Population); while not (terminal condition satisfied) do

MatingPool = reproduce(Population);

MutationPool = crossover(MatingPool);

Population $=$ mutation $($ MutationPool);

end while evaluate(Population);

end procedure

Initial population : $\quad v_{i}=\left[\begin{array}{lllll}x_{1} & x_{2} & x_{3} & \cdots & x_{30}\end{array}\right], i=50$

Evaluation :

$$
\left.\operatorname{Eval}\left(v_{i}\right)=f\left(x^{i}\right), i=1,2, \cdots, 50\right)
$$

$$
F=\sum_{i=1}^{50} \operatorname{Eval}\left(v_{i}\right)
$$

Select :

$$
\begin{aligned}
p_{i} & =\frac{\operatorname{Eval}\left(v_{i}\right)}{F}, i=1,2, \cdots, 50 \\
q_{i} & =\sum_{j=1}^{i} p_{j}, i=1,2, \cdots, 50
\end{aligned}
$$

Crossover : $\quad p_{c}=\frac{p_{c t}}{\left(p o p_{\_} \text {size }\right)}=\frac{35}{50}=0.7$

where, $p_{c}$ is the probability of breeding, $p_{c t}$ is the number of genes that is the breeding target. 
Mutation : $\quad p_{m}=\frac{p_{m t}}{\left(p o p_{\_} \text {size }\right)}=\frac{5}{50}=0.1$

where, $p_{m}$ is the probability of mutation, $p_{m t}$ is the number of genes that is the mutation target

End : $\quad S T O P>G, G=100$

We established the PLC network for comparing simulation data with the actual PLC noise pattern. (Fig. 3(a)) The DCU at the left bottom is connected to the PLC modem and spectrum analyzer at the right bottom by power line. Also, the PLC modem is connected to the laptop by RJ45 port for data transmission. The obtained frequency image is shown in Fig. 3(b)

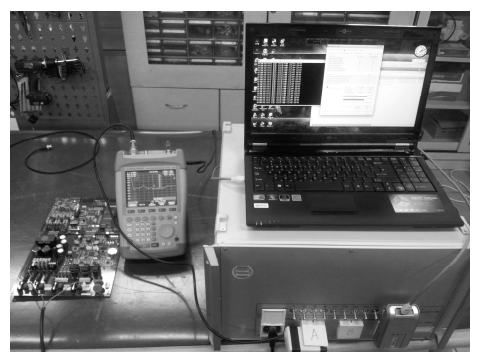

(a) The PLC network for experiment

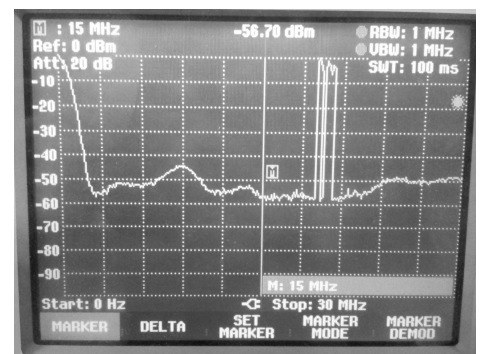

(b) The PLC spectrum pattern

Fig. 3. Lab environment and frequency pattern image

The remote PLC modem connected to the DCU transmits RF signal strength to a remote server by scanning $1 \sim 30 \mathrm{MHz}$ frequency band per $1 \mathrm{MHz}$ regularly to provide the digital data of specific RF signal strength to the simulator for pattern recognition. The simulator on a remote server predicts continuous frequency waveform by performing GA with the data and it discriminates the particular signal and color noise.

\section{Performance and result of the noise pattern recognition}

We applied GA for pattern recognition of the PLC color noise. We predicted and recognized the overall pattern with dispersed data of a particular frequency by measuring the entire PLC communication frequency band. As a result, we were approximately able to estimate the pattern of entire frequency and isolate the color noise occurred at a specific frequency from a signal. The approximately predicted graph is shown in Fig. 4. The trianlge shape means noise, circle shape means signal in the Fig. 4. We drawed a change in the pattern by increasing population size and generation as shown in Fig. 5. As a result, we make sure that the overall pattern is remained constantly but the slight change.

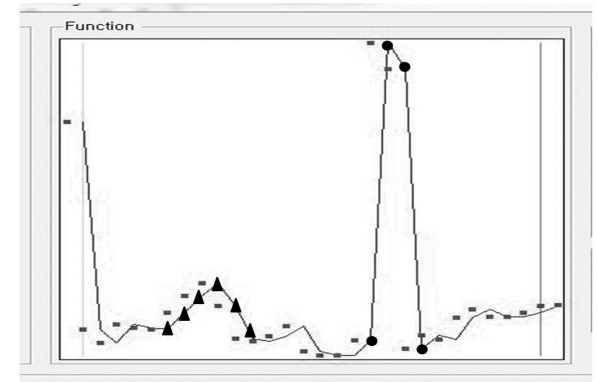

Fig. 4 The characteristic pattern recognition of the PLC after applying algorithm.

It is essential to ensure quality and reliability of the PLC communication which has come to the main problem of the AMR-based AMI system. Consequently, we studied the noise pattern recognition as the pre-required base technology for applying other technology to reduce and eliminate the noise of PLC. We simulated only for the color noise occurs in a specific frequency. However, the different types of noise can occur in PLC at the same time and it causes complex problems. Therefore, the research for the white noise across the whole spectrum and other types of noise is necessary in the future. The $\mathrm{H} / \mathrm{W}$ processor for implementing the $\mathrm{S} / \mathrm{W}$ simulation should be developed to recognize the pattern during unstable communication status due to noise and also take the measures for it in real-time.[7, 8, 9] 


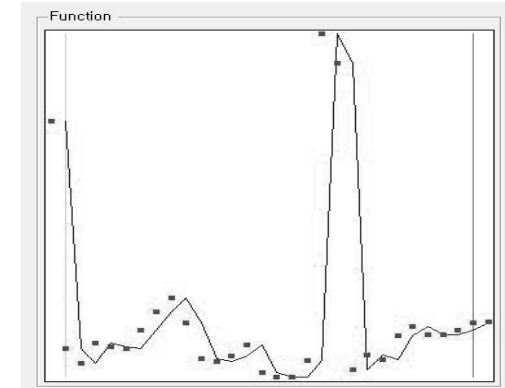

(a) size $=50$, Iteration $=100$

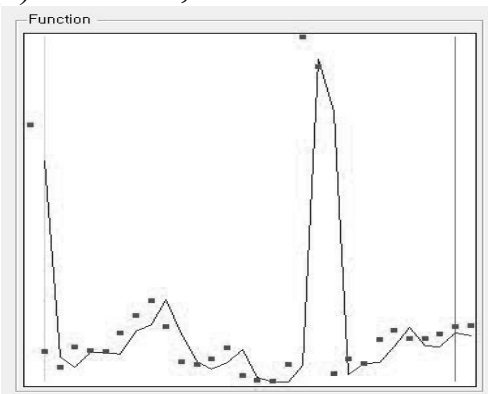

(d) size $=50$, Iteration $=1000$

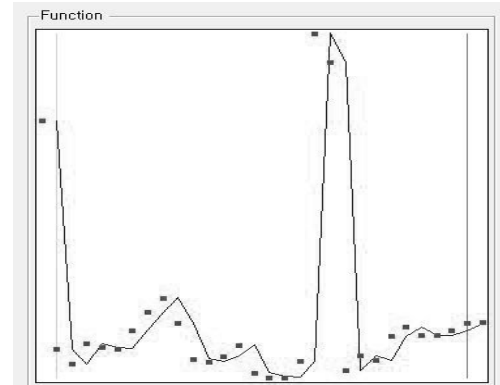

(b) size $=70$, Iteration $=100$

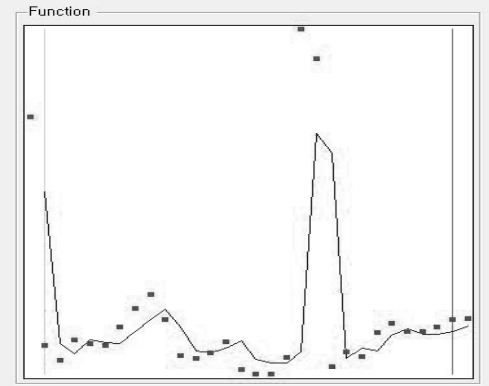

(e) size $=70$, Iteration $=1000$

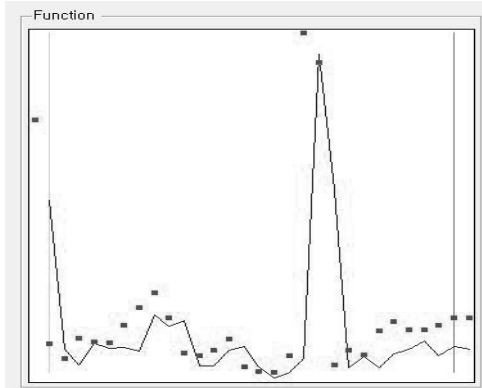

(c) size $=90$, Iteration $=100$

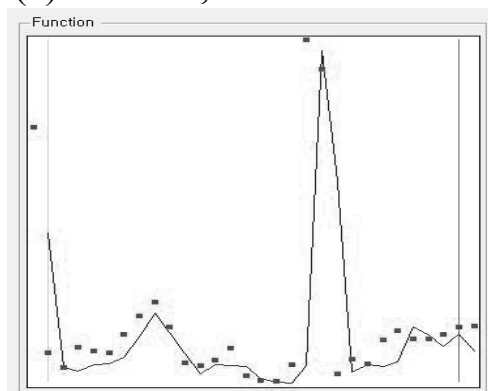

(f) size $=90$, Iteration $=1000$

Fig. 5 Simulated pattern by changing population size and generation step

\section{Conclusion}

In this paper, we applied GA for color noise cancelation and noise analysis of the transmitted signal. The addictive noise in transmitted signal is efficiently separated with GA. The analyzed noise information can be used for noise cancelation in power line communication. With this approach, we can remove the noise problems in PLC and improve the stability of system and the reliability of received data. We clarify that we developed the simulator mentioned in this paper by modifying the base program code following "GNU General Public License, version3 (GPL-3.0)". [10]

\section{Reference}

[1] Park, B.S, Hyun, D.H, Cho, S.K. "Implementation of AMR system using power line communication". Transmission and Distribution Conference and Exhibition 2002: Asia Pacific. IEEE/PES, Vol. 1, Oct. 2002, pp. 18-21

[2] Intemational Organization for Standardization, Information technology -Telecommunications and information exchange between systems - Power Line communication (PLC) - High speed PLC medium access control (MAC) and physical layer (PHY), ISO/IEC 12139-1,(2009)

[3] Yong-tao Ma, Kai-hua Liu,. "Implementation Modeling the Colored Background Noise of Power Line Communication Channel Based on Artificial Neural Network". 978-1-4244-7596-4/10, IEEE 2010

[4] Jin-jeong kim, duck-jin Chung, "Implementation of Genetic Algorithm Processor based on Hardware Optimization for Evolvable Hardware", KIEE 49D-3, 2000

[5] sung-su park, hae-young park, "Genetic Algorithm is coded by C++", Han All, pp 3-18, 2001

[6] Sankar K. Pal, Paul P. Wang, "Genetic algorithms for Pattern Recognition", ISBN 0-8493-9467-8, CRC Press, Inc. pp 4, 1996

[7] suk-woo jeong, hyun-sik kim, dong-sun kim, duck-jin kim, "Implementation of Adaptive Genetic Algorithm Processor for Evolvable Hardware”, KIEE 53D-4, 2004

[8] Barry Shackleford et al., "A High-Performance Hardware Implementation of a Survival-Based Genetic Algorithm”, ICONIP’97 pp. 686-691., Nov. 1997

[9] N. Yoshida, T. Moriki and T. Yasuoka, "GAP: Genetic VLSI processor for genetic algorithm", Second International ICSC Symp. On Soft Computing, pp. 341-354., 1997

[10] Andrew Kirillov, "Time Series Prediction", 16 Oct 2006, United Kingdom 